\title{
From Capsule to Helix: Guest-Induced Superstructures of Chiral Macrocycle Crystals
}

\author{
Avishek Dey ${ }^{1}$, Santanu Chand ${ }^{1}$, Lukman O. Alimi ${ }^{1}$, Munmun Ghosh ${ }^{1}$, Luigi Cavallo² and Niveen M. \\ Khashab $^{1 *}$ \\ ${ }^{1}$ Smart Hybrid Materials Laboratory (SHMs), Advanced Membranes and Porous Materials Center, King Abdullah University \\ of Science and Technology (KAUST), Thuwal 23955-6900, Saudi Arabia \\ ${ }^{2}$ King Abdullah University of Science and Technology (KAUST) KAUST Catalysis Center (KCC), Thuwal 23955-6900, \\ Saudi Arabia
}

\begin{abstract}
The prediction, control, understanding and elucidation of phase transition from gel to crystal is in high demand for the development of various functional materials with macroscopic properties. Here, we show a detailed and systematic description of the self-assembly process of enantiopure trianglimine macrocyclic host from gel to single crystals. This proceeds via an unprecedented formation of capsule-like or right-handed helix superstructures as metastable products, depending on the nature of the guest molecule. Mesitylene promotes the formation of capsule-like superstructures; whereas, toluene results in the formation of helices as intermediates during the course of crystallization. Single crystal results demonstrate that the crystals obtained via the direct selfassembly from the gel phase are different from the crystals obtained from the stepwise assembly of the intermediate superstructures. Hence, investigating the phase-transition superstructures that self-assemble through the process of crystallization can unravel new molecular ordering with unexplored host-guest interactions. Such understanding will provide further tools to control hierarchical assemblies at the molecular level and consequently design or dictate the properties of evolved materials.
\end{abstract}

\section{INTRODUCTION}

Predicting the molecular self-assembly of the simplest crystalline structure represents a challenge of the highest scientific and technological importance. Crystals, under the right circumstances, could be thought of as the sum of a series of molecular recognition events, self-assembly, rather than the result of the need to "avoid a vacuum". ${ }^{1}$ In this context, crystals might be regarded as being single chemical entities and as such are perhaps the ultimate examples of supramolecular

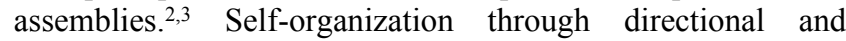
complementary noncovalent interactions, such as hydrogen bonds and metal coordination, underlies crystal engineering to achieve the most favorable molecular arrangements during the course of crystallization. ${ }^{4}$ Moreover, solvent or guest dictated self-organization of the host molecules plays a pivotal role in the final crystal assembly using the molecular recognition approach..$^{5-10}$ The visualization of the transiently trapped guest molecule in the crystalline nanochannel of ordered frameworks was recently demonstrated showcasing the effect of hydrogen bonding and $\pi \cdots \pi$ interactions on crystal packing. ${ }^{11,12}$ Other seminal work on porous molecular materials such as organic cages showed a critical correlation between the crystal structure and the nature of guest molecules. ${ }^{13-15}$ Engineering of nonporous crystalline materials such as nonporous adaptive crystals (NACs) showed that the crystal structure transformation is induced by a preferable guest molecule. ${ }^{16,17}$ Furthermore, guest templated assembly promotes the formation of evolved superstructures as elegantly demonstrated in the construction of supramolecular nanotubes from the enantiomeric naphthalenediimide (NDI) macrocycles and the porous molecular 1D nanotubes and 3D networks from organic cages. ${ }^{18,19}$

The inclusion of liquid and gas molecules as guests into a solid matrix is well known, however the mechanism of such assembly leading to the corresponding crystals formation at the molecular level is poorly understood. Such insight can guide researchers to control assemblies with specific guests to make materials with superior properties. Molecular recognition of guest molecules has been extensively studied using different host macrocycles. ${ }^{16,20-21}$ The guest uptake can take place via two routes: (a) guest uptake into the pores by diffusion and (b) guest binding to the binding pocket of the inner pore via adsorption.

Here, we studied the stepwise self-assembly process of enantiopure trianglimine macrocycles in the presence of different guests where metastable capsule-like or helical superstructures were obtained prior to the growth of the crystalline materials (Figure 1). As the energy-saving separation of benzene isomers is of prime industrial interest, we designed our imine macrocycle with an intrinsic pore that can host such guest molecules (Figure 2a). ${ }^{21,22}$ Intermolecular C$\mathrm{H} \cdots \pi$ interactions between the host and guest facilitates the molecular recognition and the eventual binding. Furthermore, chirality plays a major role in dictating the final assembly as previously reported. ${ }^{18}$ The entire process takes place under thermodynamic control similar to host-guest complexation in solution where the geometry of the guests are well equilibrated and ordered like porous complexes. ${ }^{23}$ We envisage that this work will promote the exploration of other superstructures that assemble during crystallization, which can lead to uncharted molecular ordering and ultimately to unique materials with new properties. 


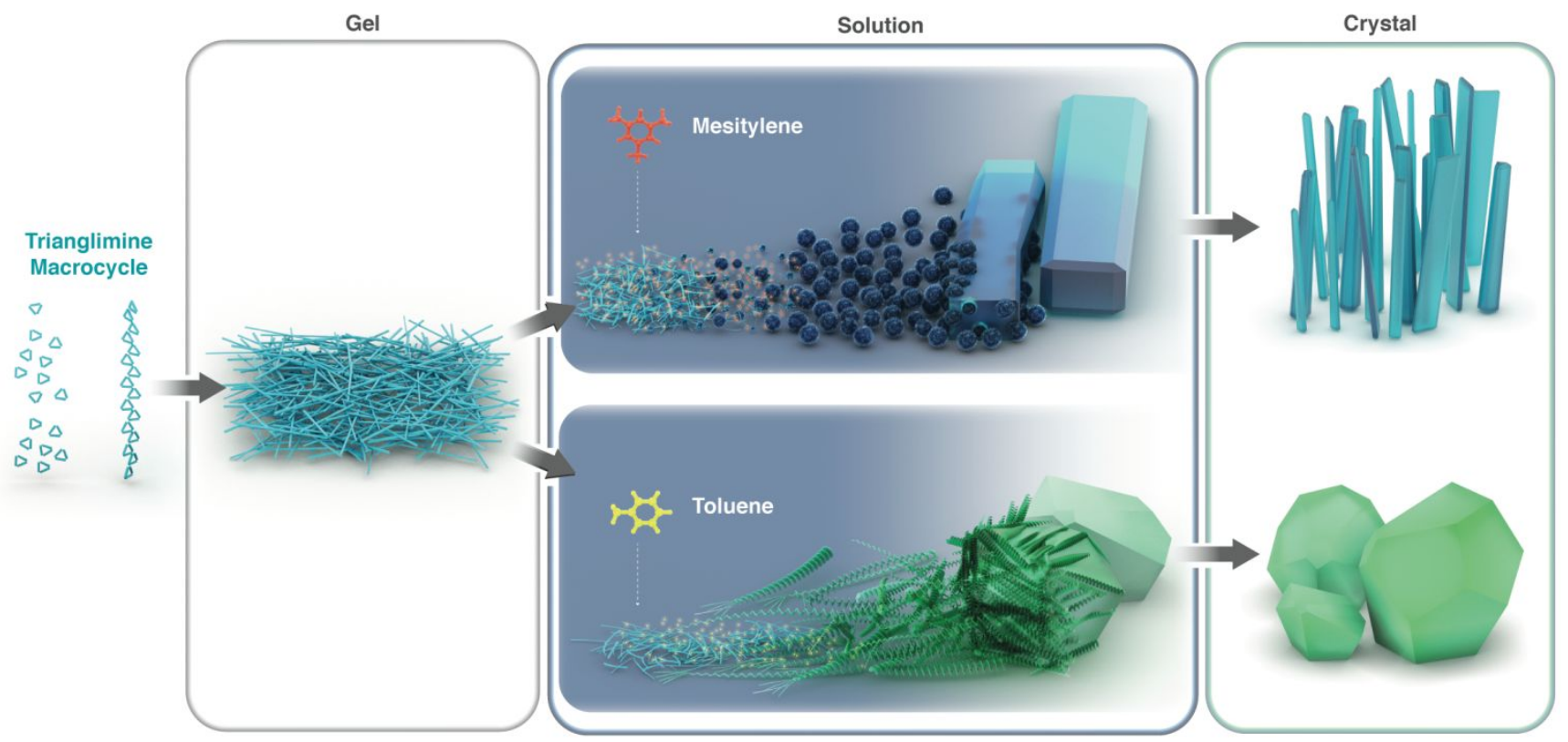

Figure 1. Stepwise guest-induced self-assembly of trianglimine macrocycle crystals.

\section{RESULTS AND DISCUSSION}

Chiral trianglimine macrocycle $\mathbf{1}$ was synthesized according to previous reports ${ }^{24-30}$ by reacting $(1 R, 2 R)$-diaminocyclohexane $(0.275 \mathrm{~g}, 2.38 \mathrm{mmol})$ with 4,4'-diformylbiphenyl $(0.5 \mathrm{~g}, 2.38$ $\mathrm{mmol})$ in $\mathrm{CHCl}_{3} /$ methanol $(1: 10)$ in the presence of triethylamine with $90 \%$ yield (Figure S1). ${ }^{1} \mathrm{H}$ and ${ }^{13} \mathrm{C}$ NMR confirm the formation of the [3+3] trianglimine as a pure product (Figure S2 and Figure S3). High resolution mass spectrometry showed a molecular ion peak at $\mathrm{m} / \mathrm{z} 865.5091$ for $[\mathrm{M}+\mathrm{H}]$, which is in agreement with the molecular weight of the $[3+3]$ condensation product (Figure S4). Macrocycle $\mathbf{1}$ is designed with an intrinsic pore diameter of $12 \AA$ which can be ideal for the recognition of benzene isomers (Figure 2a). Unlike trianglamine, which consist of a flexible skeleton, trianglimine is generally shape persistent, and rarely display various types of conformations in the solid state because of its rigid skeleton. Although, the stability of the imine bond in aqueous solution is still an enigma to researchers, in our case, the stability of macrocycle $\mathbf{1}$ was confirmed in water as illustrated in Figure S5.

Attempts to crystalize $\mathbf{1}$ in ethyl acetate (EA) resulted in the formation of octahedron shaped crystals (1A) which is suitable for single crystal X-ray diffraction (Figure 2b and Figure S6). Whereas, it crystallizes in needle shaped guest-free crystals (1B) from chloroform $\left(\mathrm{CHCl}_{3}\right)$ in EA (2:1) in hexane vapor (Figure 2c and Figure S6). Interestingly, we found that $\mathbf{1}$ in $\mathrm{CHCl}_{3}$ /EA (4:2) resulted in the formation of a supramolecular organogel which consists of fibrous assembly as observed under SEM (Figure S6). The single crystal analysis of $\mathbf{1 A}$ reveals that it crystalizes in a triclinic crystal system with chiral $P 1$ space group and the asymmetric unit contains four units of trianglimine and two units of EA (Figure 3a). Two trianglimine units at the center are eclipsed to each other and the other two trianglimine units are perpendicular to the two eclipsed units on either side when viewed along the crystallographic $a$ axis. It possesses window to window arrangements in the $c$ axis where the EA molecules sit perfectly inside the intrinsic cavity of the

trianglimine and are stabilized by $\mathrm{C}-\mathrm{H} \cdots \mathrm{O}$ interactions $(2.535$ $\AA$ ) between the $\mathrm{O}-\mathrm{C}=\mathrm{O}$ of EA with the biphenyl moieties (Figure $2 b$ ). The crystal packing shows that molecules form interconnected layered structure where the channels are being filled up with EA (Figure 3b). The host layers are interconnected with each other by $\pi \cdots \pi$ interactions $(3.845 \AA)$ between the biphenyl moieties (Figure 3c). The host trianglimines are arranged in a head to tail fashion to create the interconnected layers which are responsible for the channels (Figure 3b).

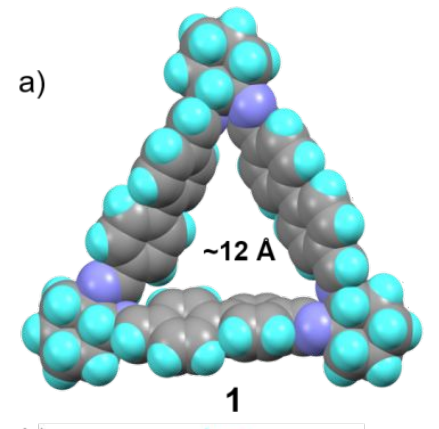

b)

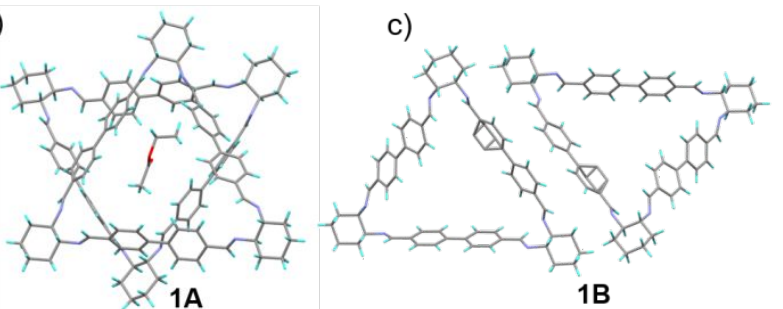

Figure 2. (a) Space filling representation of the crystalline trianglimine 1 to host potential benzene derivatives. (b) Crystal structure of 1A shows window to window arrangement of trianglimines where EA sits inside the intrinsic cavity. (c) Crystal structure of 1B shows the perpendicular arrangements of trianglimines. 
The guest accessible volume per unit cell for $\mathbf{1 A}$ is approximately $810 \AA^{3}$, which is close to $14.5 \%$ of the unit cell. SCXRD analysis of $\mathbf{1 B}$ reveals that it crystallizes in monoclinic system bearing the space group $P 2_{1}$ and the asymmetric unit contains only one unit of trianglimine. The structural analysis also reveals that two trianglimines are nearly perpendicular to each other and this makes the arrangement window to vertex along $b$ plane (Figure 2c). The triangles are stacked in an eclipsed manner through head-tail fashion in $b$ plane. The interlayer distance between two trianglimine molecular interior is approx. $4.2 \AA$. Such type of arrangement contracts the unit cell creating less space for the guest molecules. The packing of the molecules shows that it has layered structure with channels (Figure 3d). The guest accessible volume per unit cell is $666 \AA^{3}$, which is close to $25.6 \%$ of the unit cell volume.
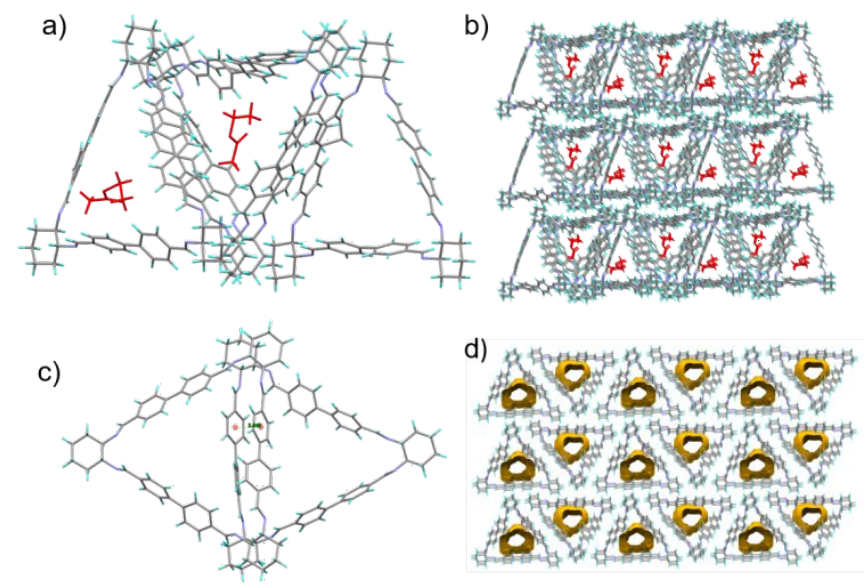

Figure 3. Description of the crystal structure of 1A and 1B: (a) Single crystal structure depicts how the EA intercalates with host in the asymmetric unit. (b) Packing of the layered structure of 1A containing channels where EA molecules are occupied. (c) Two trianglimine macrocycles are extended by $\pi \cdots \pi$ interaction in $\mathbf{1 A}$. (d) Packing of the layered structure of 1B containing empty channels.

Since 1 accommodates EA solvent molecules inside its intrinsic cavity, we then resorted to crystalize it in the presence of mesitylene as an aromatic guest (best fit for the intrinsic pore). Initial attempts to crystalize 1 in $\mathrm{CHCl}_{3} / \mathrm{EA} /$ mesitylene $(4: 2: 1)$ resulted in the formation of supramolecular organic gel (Figure S7). The organogel dissolved to a solution at room temperature after 14 days from which needle-shaped crystals 1C were obtained. Addition of the mesitylene to the gel obtained from 1 in $\mathrm{CHCl}_{3} / \mathrm{EA}$ also resulted in the same observation. To understand the mechanism of the gel formation as well as the phase transition from gel to crystals, we first carried out a series of SEM studies. SEM images of the gel show that $\mathbf{1}$ assembles to form a fibrous material (Figure 4). After the addition of the mesitylene guest molecules, the fibers are converted to capsules- like superstructures in solution, which we believe is due to the inclusion of the guest molecules (Figure 4 , top). The dynamic light scattering (DLS) studies show that the average size of the capsule is $1 \mu \mathrm{m}$ and they have a negative zeta potential. We hypothize that the capsule-like superstructures are formed as a metastable state under kinetic control and are subsequently converted to the thermodynamically stable needle-shaped crystals (Figure S8).The $\mathrm{C}_{3}$ symmetry of mesitylene also induces the crystallization in a high symmetry space group. ${ }^{31}$ Furthermore, we believe that the slow nucleation process is responsible for the formation of these capsule-like superstructures. This was further verified by heating the gel to $90^{\circ} \mathrm{C}$, which resulted in the direct formation of a fibrous assembly prior to crystallization without the formation of the capsule-like superstructures (Figure S9). The phase change of the gel was verified by differential scanning calorimetry (DSC) where a clear phase transition was seen in the gel state (Figure S10). The crystal structure analysis of $\mathbf{1 C}$ reveals that it crystallizes in highly symmetric hexagonal crystal system with $P 6_{1}$ space group and the asymmetric unit contains one unit of host trianglimine and two units of mesitylene guest where host/guest ratio is 1:2 (Figure 5a). The structural analysis also reveals that it possesses window to vertex arrangement where trianglimines are perpendicular to each other. One mesitylene perfectly sits inside the intrinsic cavity of the trianglimine which is being stabilized by $\mathrm{C}-\mathrm{H} \cdots \pi$ interactions $(2.690 \AA)$ between methyl group of the guest and the centroid of the biphenyl systems in the host macrocycle (Figure $5 \mathrm{~b}$ and Figure $5 \mathrm{c}$ ). The other one is present in the interstitial position. When viewed along $c$ axis, the crystal packing shows that the guest molecule between the three macrocyclic molecules (interstice) undergoing $\mathrm{C}-\mathrm{H} \cdots \pi$ interactions $(2.875 \AA)$ (Figure $5 \mathrm{~d}$ and Figure S11). It is shown that the guest molecules present outside the cavity are also stabilized by $\mathrm{C}-\mathrm{H} \cdots \pi$ interactions $(2.875 \AA)$ which is actually responsible for the changes in the torsional angles of the biphenyl systems in the macrocycle. The cyclohexyl group also contributes in directing the inclusion of the guest molecule in the interstice between the three macrocyclic molecules through $\mathrm{C}-\mathrm{H} \cdots \pi$ interactions (Figure S11a). The macrocyclic molecules exist in form of layers when viewed along the $b$ axis. Each layer is connected through $\mathrm{C}-\mathrm{H} \cdots \pi$ interactions to form a $2 \mathrm{D}$-network (Figure 5e). In other words, the entire crystal network is built by a $2 \mathrm{D}$-network of $\mathrm{C}-\mathrm{H} \cdots \pi$ interactions. Although these interactions are weak, their high directionality contributes immensely to the assembly of these molecules. Apart from C$\mathrm{H}^{\cdots} \pi$ interactions, two consecutive host bilayers are connected by weak $\pi \cdots \pi$ interaction of $4.338 \AA$. The guest accessible volume per unit cell is $2809 \AA^{3}$, which means $28.5 \%$ of the volume is available for the inclusion of mesitylene. 


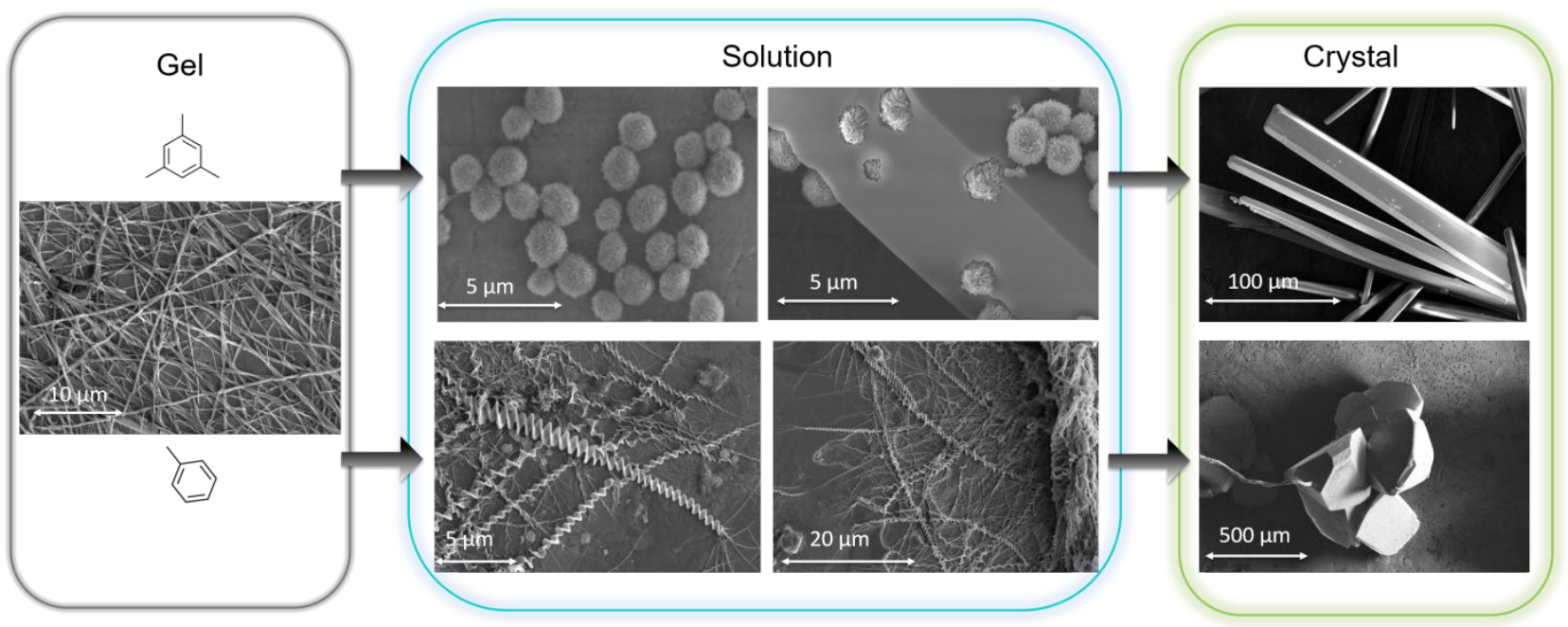

Figure 4. SEM images of the self-assembly process of 1 from gel (fibers) to solution to crystals depending on the guest molecules, mesitylene (top) and toluene (bottom).

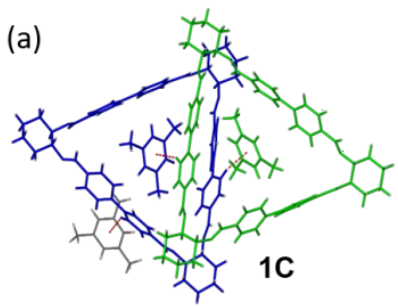

(b)

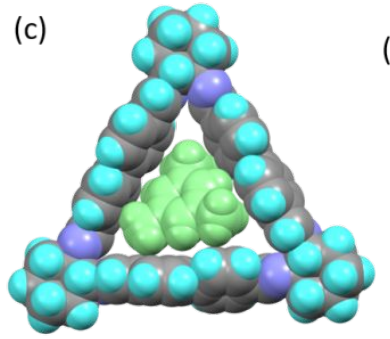

(d)

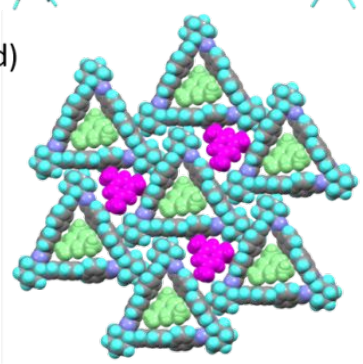

(e)

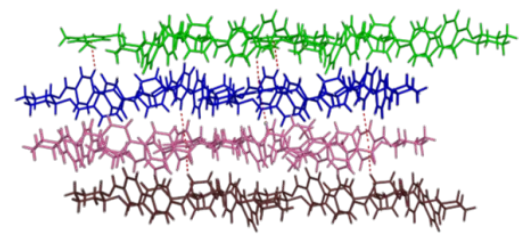

Figure 5. Host-guest interactions from SCXRD. (a) Crystal structure of $\mathbf{1 C}$ shows mesitylene intercalating with host $\mathbf{1}$. (b) Mesitylene is stabilized in the macrocyclic host by $\mathrm{C}-\mathrm{H} \cdots \pi$ interaction. (c) Space filling structure shows how mesitylene fits in the intrinsic cavity of the host. (d) Mesitylene is occupying both internal cavity and interstitial space of the macrocycle via $\mathrm{C}-\mathrm{H} \cdots \pi$ interaction (1:2 ratio). (e) Two dimensional arrangements of the macrocycle layers where they are connected by weak $\pi \ldots \pi$ interactions when viewed along $b$ axis.

To better understand the role of guest molecules in the stepwise assembly, $\mathbf{1}$ was crystalized in the presence of toluene, another aromatic guest, in $\mathrm{CHCl}_{3} / \mathrm{EA} /$ toluene $(4: 2: 1)$. As expected, a fibrous gel was obtained and went through a selfassembly process. Interestingly, this guest-prompted assembly did not yield a capsule-like superstructure but rather a metastable right-handed helix before forming the stable crystals 1D (Figure 4, down). Similar to mesitylene, addition of the toluene directly to the gel obtained from $\mathbf{1}$ in $\mathrm{CHCl}_{3} / \mathrm{EA}$ also resulted in the same observation yielding crystals 1D. The size of the helices is $500-700 \mathrm{~nm}$ in diameter and they are few micrometers long with a uniform helical pitch of approximately $1 \mu \mathrm{m}$ (Figure 4 and Figure S12). PXRD studies were conducted to verify the phase purity of the obtained crystals (Figure S13). To confirm the helical nature of the ribbons, circular dichroism (CD) spectra of the host macrocycle $\mathbf{1}$ with toluene and $\mathbf{1}$ in $\mathrm{CHCl}_{3}$ were measured at room temperature. $\mathrm{CD}$ spectra confirm that the helical ribbons are formed with right handedness in case of toluene at that particular concentration and state. The CD spectrum of 1 in $\mathrm{CHCl}_{3}$ exhibits positive signals at around 323 $\mathrm{nm}$ which can be assigned to the imine $\mathrm{n}-\pi^{*}$ transition. On the other hand, CD of toluene included host exhibits both positive and negative signals at 319 and $268 \mathrm{~nm}$ respectively with zero crossing close to $290 \mathrm{~nm}$, which indicates the transfer of chiral information of the self-assembled product in a helical sense (Figure S14). The single crystal structural analysis revealed that it crystallizes in triclinic $P 1$ space group and contains four units of trianglimine and four units of toluene (Figure 6a). This indicates that host/guest ratio is maintained $1: 1$ in the crystal lattice. In this scenario, toluene perfectly fits into the host trianglimine where host-guest interaction is being stabilized by $\mathrm{C}-\mathrm{H} \cdots \pi$ interactions of the methyl group (Figure $6 \mathrm{~b}$ and Figure $6 c)$. Two trianglimines are in overlapping position, whereas the other two are placed sidewise in head-to-tail fashion. This type of arrangement makes them window to vertex, where guest molecules are placed in the intrinsic cavity created by this arrangement. The packing diagram suggests that it has a layered structure where toluene is present inside the connecting channels (Figure 6d). The diffusion of guest molecules also influences the changes in torsional angle in the biphenyl systems present in the macrocycle. PLATON analysis reveals the solvent accessible volume per unit cell is $1665 \AA^{3}$, which means $30.3 \%$ of the volume is available for the inclusion of toluene. 

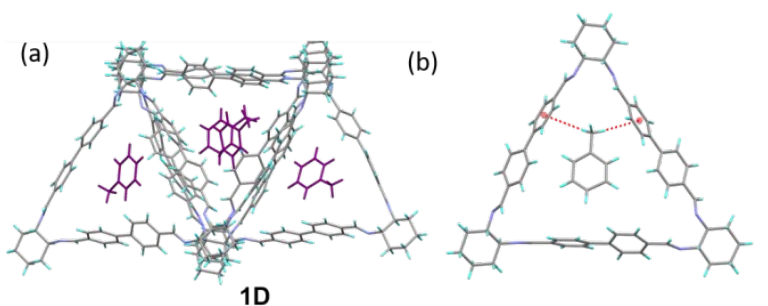

(c)

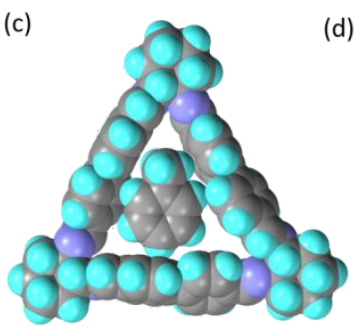

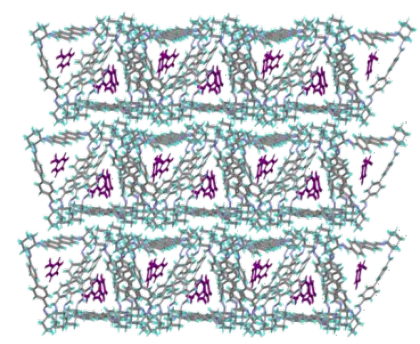

Figure 6. (a) Crystal structure of 1D shows toluene intercalating with host 1 in 1:1 ratio. (b) Toluene is stabilized in the host by C$\mathrm{H} \cdots \pi$ interactions. (c) Space filling structure shows how the guest fits in the intrinsic cavity. (d) Overall packing of the host-guest structure.

We propose that studying the self-assembly process and identifying the intermediate superstructures, which are formed through the crystallization process, may lead to new molecular ordering and consequently allows the re-engineering of

(a)

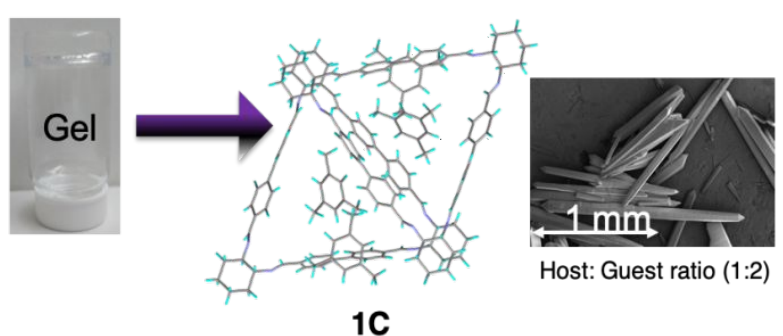

(c)

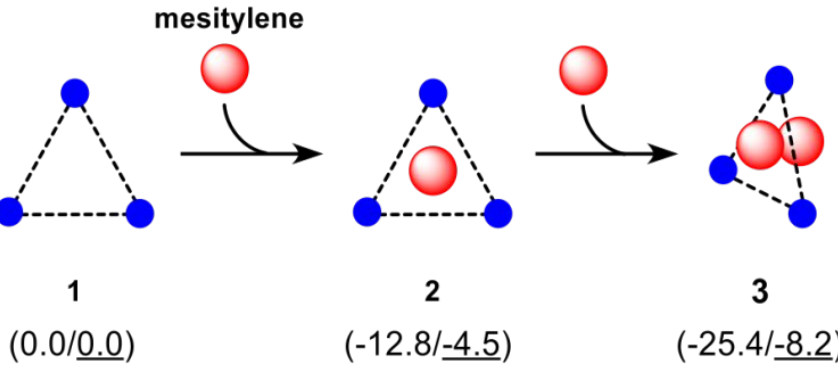

materials properties. To best test this hypothesis, we collected two crystals separately: one obtained directly from the fibrous gel (after 24 days) (1C) (Figure 7a) and the other obtained from the isolated metastable capsule-like superstructures upon further addition of dichloromethane/acetonitrile (2:1) (1E) (Figure $7 \mathrm{~b}$ and S15). Single crystal analysis revealed that the host/guest ratio of the crystal obtained from the kinetic capsulelike superstructures was different from the thermodynamically stable needle-shaped crystal obtained directly from the gel (1:0.5 vs $1: 2)$. DFT calculations were performed to estimate the host-guest interaction energy for a possible kinetic versus thermodynamic pathway to form the final crystals. A unimolecular interaction results in $\mathbf{2}$ (considered as intermediate assembly), which is $-4.5 \mathrm{kcal} / \mathrm{mol}$ lower in energy compared to the free molecular form (Figure 7c). The interaction of a second mesitylene molecule with $\mathbf{2}$ leads to $\mathbf{3}$, an exergonic step by $3.7 \mathrm{kcal} / \mathrm{mol}$ (Figure 7c). This supports the thermodynamic feasibility to host two guest molecules by one trianglimine macrocycle. Analysis of the electron density ${ }^{32}$ calculations confirms the expected $\mathrm{C}-\mathrm{H} \cdots \quad \pi$ interactions occurring between 1 and mesitylene (Figure 7d and Figure S16). Moreover, PXRD analysis of the gel and the capsule-like superstructures confirmed the phase purity and the structural resemblance to the simulated patterns of the crystal structures (Figure S17).

(b)

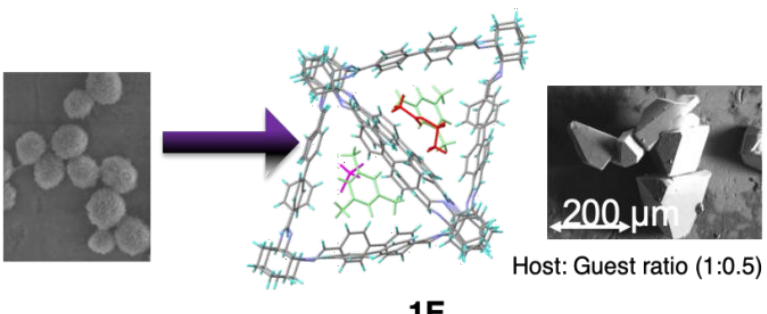

(d)

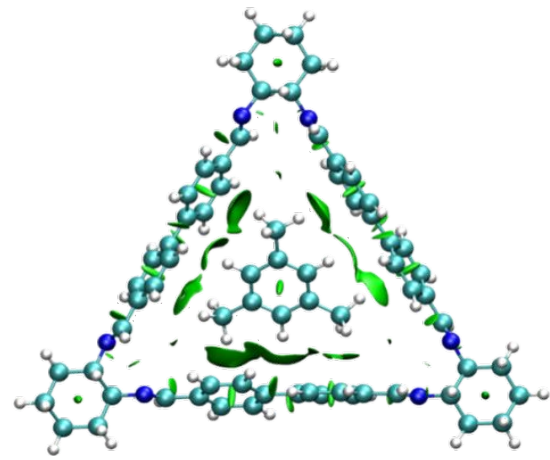

Figure 7. (a) Crystal obtained from gel via direct self-assembly (1C) vs (b) crystal obtained from the capsule-like superstructures via stepwise self-assembly (1E) at the same conditions. (c) Energy values $\left(\Delta H_{298} \mathrm{~s} / \underline{G}_{298} \mathrm{~s}\right)$ are in $\mathrm{kcal} / \mathrm{mol}$ at $\omega \mathrm{B} 97 \mathrm{xD}$ (SMDmesitylene)/Def2-TZVP//PBE0-D3/Def2-SVP level of theory. (d) Non-covalent interactions analysis of 2: large green areas between 1 and mesitylene indicate regions of weak noncovalent interactions. 


\section{Conclusions}

We have demonstrated that enantiopure trianglimine macrocycles undergo a guest-driven self-assembly process going from a gel to capsule-like or right-handed helix superstructures in solution before reassembling further to the thermodynamically stable crystalline products. The capsulelike superstructures were formed with mesitylene guest molecules, while the right-handed helices were obtained with toluene guest molecules. Host-guest ratio and $\mathrm{C}-\mathrm{H} \cdots \pi$ interactions are found to play the major role in the superstructure formation and phase transition. An alteration is observed in the host-guest ratio of the crystals obtained from the isolated metastable capsule-like superstructures (kinetic pathway) compared to the crystals obtained directly from the gel (thermodynamic pathway). This implies that a different structural arrangement can be achieved starting from the intermediate superstructures versus the original direct assembly under similar conditions. The thermodynamic feasibility of this process was further supported by DFT calculations. We believe that this work capitalizes on the importance of understanding the self-assembly process during crystallization as a plethora of kinetic superstructures can be isolated, which can be further manipulated in the presence of guest molecules to produce the next generation of unconventional hierarchical smart materials.

\section{ASSOCIATED CONTENT}

\section{Supporting Information}

Detailed synthetic procedure and characterization including NMR, Mass, SCXRD data, crystallographic figure and parameters table, SEM images of gel to crystals and PXRD, Computational results and DSC etc.

\section{AUTHOR INFORMATION}

\section{Corresponding Author}

Niveen M. Khashab - King Abdullah University of Science and Technology (KAUST), Thuwal, Saudi Arabia.

Email: niveen.khashab@kaust.edu.sa

\section{Author Contributions}

The manuscript was written through contributions of all authors. All authors have given approval to the final version of the manuscript.

\section{ACKNOWLEDGMENT}

This work was supported by King Abdullah University of Science and Technology (KAUST). We are thankful to Dr. Bholanath Maity, KAUST Catalysis Center (KCC) for fruitful discussions on theoretical calculations and studies.

\section{ABBREVIATIONS}

EA, Ethyl Acetate; DCM, Dichloromethane; ACN, acetonitrile.

\section{REFERENCES}

1) Moulton, B.; Zaworotko, M. J. From Molecules to Crystal Engineering: Supramolecular Isomerism and Polymorphism in Network Solids. Chem. Rev. 2001, 101, 1629-1658.

2) Desiraju, G. R. Crystal Engineering: From Molecule to Crystal. J. Am. Chem. Soc. 2013, 135, 9952-9967.

3) Atwood, J. L.; Barbour, L. J.; Jerga, A.; Schottel, B. L. Guest Transport in a Nonporous Organic Solid via Dynamic van der Waals Cooperativity. Science 2002, 298, 1000-1002

4) Kobayashi, K.; Sato, A.; Sakamoto, S.; Yamaguchi, K. Solvent-Induced Polymorphism of Three-Dimensional
Hydrogen-Bonded Networks of Hexakis(4carbamoylphenyl)benzene. J. Am. Chem. Soc. 2003, 125 , 3035-3045.

5) Lehn, J. M. Toward complex matter: Supramolecular chemistry and self-organization. Proc. Natl. Acad. Sci. USA 2002, 99, 4763-4768.

6) Dobrzańska, L.; Lloyd, G. O.; Esterhuysen, C.; Barbour, L. J. Guest-Induced Conformational Switching in a Single Crystal. Angew. Chem. Int. Ed. 2006, 45, 5856-5859.

7) Braga, D. Crystal engineering, Where from? Where to? Chem. Commun. 2003, 2751-2754.

8) Etter, M. C.; Urbanczyk-Lipkowska, Z.; Jahn, D. A.; Frye, J. S. Solid-State Structural Characterization of 1,3Cyclohexanedione and of a 6:1 Cyclohexanedione: Benzene Cyclamer, a Novel Host-Guest Species. J. Am. Chem. Soc. 1986, 108, 5871-5876.

9) Horike, S.; Shimomura, S.; Kitagawa, S. soft porous crystals. Nature Chemistry 2009, 1, 695-704.

10) Yang, Q. -Y.; Lama, P.; Sen, S.; Lusi, M.; Chen, K. -J.; Gao, W. -Y.; Shivanna, M.; Pham, T.; Hosono, N.; Kusaka, S.; Perry IV, J. J.; Ma, S.; Space, B.; Barbour, L. J.; Kitagawa, S.; Zaworotko, M. J. Reversible Switching between Highly Porous and Nonporous Phases of an Interpenetrated Diamondoid Coordination Network That Exhibits GateOpening at Methane Storage Pressures. Angew. Chem. Int. Ed. 2018, 57, 5684-5689.

11) Kubota, R.; Tashiro, S.; Shiro, M.; Shionoya, M. In situ Xray snapshot analysis of transient molecular adsorption in a crystalline channel. Nature Chemistry 2014, 6, 913-918.

12) Inokuma, Y.; Yoshioka, S.; Ariyoshi, J.; Arai, T.; Hitora, Y.; Takada, K.; Matsunaga, S.; Rissanen, K.; Fujita, M. X-ray analysis on the nanogram to microgram scale using porous complexes. Nature 2013, 495, 461-466.

13) Tozawa, T.; Jones, J. T. A.; Swamy, S.; Jiang, S.; Adams, D. J.; Shakespeare, S.; Clowes, R.; Bradshaw, D.; Hasell, T.; Chong, S. Y.; Tang, C.; Thompson, S.; Parker, J.; Trewin, A.; Bacsa, J.; Slawin, A. M. Z.; Steiner, A.; Cooper, A. Nature Mater. 2009, 8, 973-978.

14) Bera, S.; Dey, K.; Pal, T. K.; Halder, A.; Tothadi, S.; Karak, S.; Addicoat, M.; Banerjee. R. Porosity Switching in Polymorphic Porous Organic Cages with Exceptional Chemical Stability. Angew .Chem. Int.Ed. 2019, 58, 42434247.

15) Mastalerz, M. Porous Shape-Persistent Organic Cage Compounds of Different Size, Geometry, and Function. Acc. Chem. Res. 2018, 51, 2411-2422.

16) Jie, K.; Zhou, Y.; Li, E.; Huang, F. Nonporous Adaptive Crystals of Pillararenes. Acc. Chem. Res. 2018, 51, 9, 20642072.

17) Jie, K.; Liu, M.; Zhou, Y.; Little, M. A.; Pulido, A.; Chong, S. Y.; Stephenson, A.; Hughes, A. R.; Sakakibara, F.; Ogoshi, T.; Blanc, F.; Day, G. M.; Huang, F.; Cooper, A. I. Near-Ideal Xylene Selectivity in Adaptive Molecular Pillar[n]arene Crystals. J. Am. Chem. Soc. 2018, 140, 69216930.

18) Liu, Z.; Liu, G.; Wu, Y.; Cao, D.; Sun, J.; Schneebeli, S. T.; Nassar, M. S.; Mirkin, C. A.; Stoddart, J. F. Assembly of Supramolecular Nanotubes from Molecular Triangles and 1,2-Dihalohydrocarbon. J. Am. Chem. Soc. 2014, 136, 16651-16660.

19) Slater, A. G.; Little, M. A.; Pulido, A.; Chong, S. Y.; Holden, D.; Chen, L.; Morgan, C.; Wu, X.; Cheng, G.; Clowes, R.; Briggs, M. E.; Hasell, T.; Jelfs, K. E.; Day, G. M.; Cooper, A. I. Reticular synthesis of porous molecular 1D nanotubes and 3D networks. Nature Chemistry 2017, 9, 17-25.

20) Feynman, R. There's Plenty of Room at the Bottom. Eng Sci. 1960, 22-36.

21) Little, M. A.; Cooper, A. I. The Chemistry of Porous Organic Molecular Materials. Adv. Funct. Mater. 2020, 1909842. 
22) Zhang, G., Emwas, A.-H., Shahul Hameed, U. F., Arold, S. T., Yang, P., Chen, A., Khashab, N. M., Shape-Induced Selective Separation of Ortho-substituted Benzene Isomers Enabled by Cucurbit[7]uril Host Macrocycles. Chem, 2020, 6, 1082-1096.

23) Little, M. A.; Briggs, M. E.; Jones, J. T. A.; Schmidtmann, M.; Hasell, T.; Chong, S. Y.; Jelfs, K. E.; Chen, L.; Cooper, A. I. Trapping virtual pores by crystal retro-engineering. Nature Chemistry 2015, 7, 153-159.

24) Chaix, A.; Mouchaham, G.; Shkurenko, A.; Hoang, P.; Moosa, B.; Bhatt, P. M.; Adil, K.; Salama, K. N.; Eddaoudi, M.; Khashab, N. M. Trianglamine-Based Supramolecular Organic Framework with Permanent Intrinsic Porosity and Tunable Selectivity. J. Am. Chem. Soc. 2018, 140, 1457114575.

25) Gawroński, J.; Kołbon, H.; Kwit, M.; Katrusiak, A. Designing Large Triangular Chiral Macrocycles: Efficient $[3+3]$ Diamine-Dialdehyde Condensations Based on Conformational Bias. J. Org. Chem. 2000, 65, 5768-5773.

26) Gawronski, J.; Gawronska, K.; Grajewski, J.; Kwit, M.; Plutecka, A.; Rychlewska, U. Trianglamines-Readily Prepared, Conformationally Flexible Inclusion Forming Chiral Hexamines. Chem. Eur. J. 2006, 12, 1807-1817.

27) Kwit, M.; Skowronek, P.; Kosbon, H.; Gawronski, J. Synthesis, Structure, and Contrasting Chiroptical Properties of Large Trianglimine Macrocycles. CHIRALITY 2005, 17, S93-S100.

28) Kuhnert, N.; Straßnig, C.; Lopez-Periago, A. M. Synthesis of novel enantiomerically pure trianglimine and trianglamine macrocycles. Tetrahedron: Asymmetry, 2002, 13, 123-128.

29) Kuhnert, N.; Burzlaff, N.; Patel, C.; Lopez-Periago, A. Tuning the size of macrocyclic cavities in trianglimine macrocycles. Org. Biomol. Chem., 2005, 3, 1911-1921.

30) Kuhnert, N.; Patel, C.; Jami, F Synthesis of chiral nonracemic polyimine macrocycles fromcyclocondensation reactions of biaryl and terphenyl aromaticdicarboxaldehydes and 1R,2R-diaminocyclohexane. Tetrahedron Lett. 2005, $46,7575-7579$.

31) Thaimattam, R.; Xue, F.; Sarma, J. A. R. P.; Mak, T. C. W.; Desiraju, G. R. Inclusion Compounds of Tetrakis(4nitrophenyl)methane: $\quad \mathrm{C}-\mathrm{H} \cdots \mathrm{O} \quad$ Networks, Pseudopolymorphism, and Structural Transformations. J. Am. Chem. Soc. 2001, 123, 4432-4445.

32) Contreras-García, J.; Johnson, E. R.; Keinan, S.; Chaudret, R.; Piquemal, J.-P.; Beratan, D. N.; Yang, W. NCIPLOT: A Program for Plotting Noncovalent Interaction Regions. J. Chem. Theory Comput. 2011, 7, 625-632. 
Table of Contents

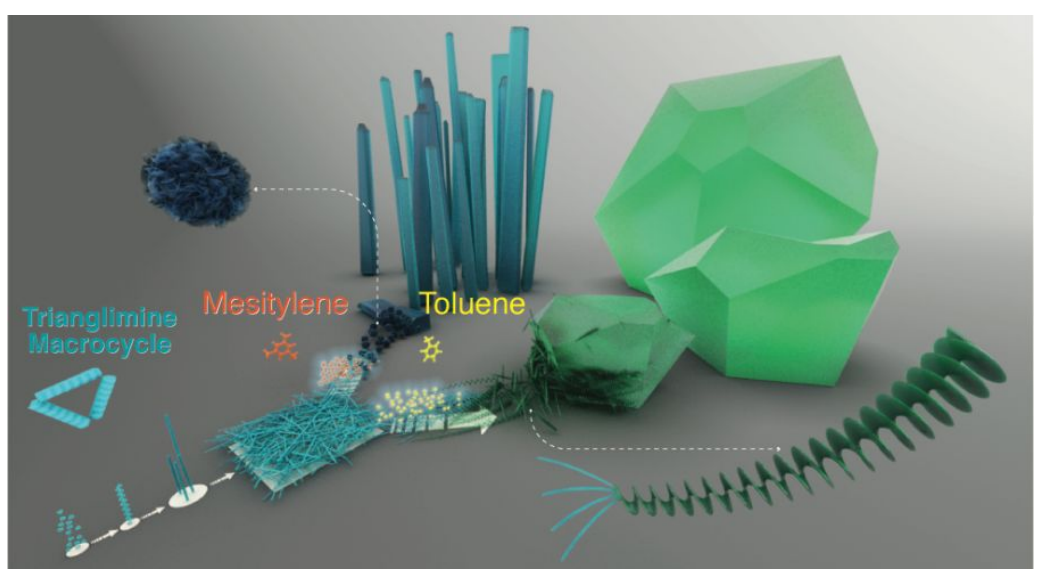

